\title{
A clinical analysis and study of 110 pediatric cases with Aminophylline poisoning
}

\author{
Fuyong Jiao ${ }^{*}$, Nan Gao ${ }^{1}$, Sheng Zhang ${ }^{1}$ and Yuhua Yang ${ }^{2}$ \\ ${ }^{1}$ Children's Hospital, Shaanxi Provincial People's Hospital, China \\ ${ }^{2}$ Xi'an Medical College, China
}

\begin{abstract}
Aminophylline is important drug for the treatment of bronchial asthma. Aminophylline by Intravenous injection is quick effect, good curative effect, but the safety margin is small, the body eliminate rate of individual difference is bigger, if use undeserved, often easy to cause serious side effects, and even death. Analysis for 110 cases of children with aminophylline poisoning were 1 year old children poisoning cases in the highest, accounted for $55.5 \%$, in 110 cases main disease is bronchiolitis (asthmatic suffocating pneumonia), the most common symptoms is nausea and vomiting, accounted for $72 \%$, maximum dose of 1500 mg . the treatment is gastric lavage, intravenous fluids, medicine for cardiac failure, diuresis and blood perfusion and hemo- dialysis etc.
\end{abstract}

\section{Introduction}

Aminophylline is an important drug for the treatment of bronchial asthma [1]. Intravenous bolus injection of aminophylline has rapid antiemetic effects and is effective in clinical practice. However, the safety range is small, the therapeutic index is narrow, and the individual elimination rate in vivo is relatively large. If used improperly, it often causes serious side effects and even life-threatening. Its pharmacological effects are mainly derived from theophylline, and ethylenediamine enhances its water solubility. The drug has a direct relaxing effect on respiratory smooth muscle [2]. The mechanism of action is relatively complex. In the past, it was thought that by inhibiting phosphodiesterase, the intracellular cAMP content was increased. Recent experiments have suggested that the bronchodilating effect of theophylline is partly due to the release of endogenous epinephrine and norepinephrine. In addition, theophylline is a purine receptor blocker that can counteract the contractile effects of adenine and other respiratory tracts. Theophylline can increase the contractility of the diaphragm, especially when the diaphragm is incapable of contraction, and is therefore beneficial to improve breathing [3-5] (Table 1).

\section{General information}

- From 1978 to 2004 Inpatient medical records:

- Male 64 (55\%); female 45 (45\%); male: female $=4: 3$.

- Age: <1-year-old 61 cases accounted for 55.5\%; 1-3 years old 31 cases accounted for $28.2 \%$; > 3 years old 18 cases accounted for $16.3 \%$.

- Primary disease: 105 cases of pneumonia and asthmatic bronchitis; 1 case of acute glomerulonephritis; 4 cases of misuse.

\section{Medication route}

- Parents fed on their own excessive aminophylline in 11 cases (4 cases were wrongly taken).

- Medical staff use drugs: a. 12 cases of oral medication b. intramuscular injection in 15 cases; intravenous infusion of 46 cases; push injection in 17 cases c. drug allergy in 8 cases d. improper drug compatibility in 1 case.

\section{Treatment}

- Gastric lavage, vomiting: General oral poisoning, gastric lavage immediately after admission, 20\% mannitol can be used to guide diarrhea.

- Rehydration, sedatives, diuretic, oxygen: all intravenous furosemide $(1 \mathrm{mg} / \mathrm{kg}$ ); large doses of vitamin C added to $5 \%$ or $10 \%$ glucose; hypoxia can be oxygen; anxious intramuscular injection Lumina (3$4 \mathrm{mg} / \mathrm{kg})$; convulsant diazepam $(0.3-0.5 \mathrm{mg} / \mathrm{kg})$.

- If there is bleeding from the digestive tract, the bleeding should be stopped immediately, and blood transfusion can be given as appropriate.

- Outcomes: Outpatient: 9 cases; Inpatient: 93 cases cured (3-8 days); Death: 8 cases, accounting for $7 \%$.

\section{Special cases}

\section{Male, 13 years old, medical history}

The patient mistakenly took 15 tablets of aminophylline $(0.1 \mathrm{~g} /$ tablet) (1500 $\mathrm{mg}$ in total) at one time, and developed chest discomfort, nausea, vomiting, and excitement at 4 hours after taking the drug. Urgent treatment at a local hospital, given gastric lavage, oxygen, intravenous infusion of "mannitol, pantoprazole, potassium chloride, amiodarone" and other drug treatment, the symptoms have eased. 3 Suddenly appeared pale, sweating, numbness in hands and feet, discomfort in the precordial area, examination: body temperature:

${ }^{\star}$ Correspondence to: Jiao F, Prof. and Head of Children's Hospital, Xi'an Jiaotong University, Shaanxi Provincial People’s Hospital, China, E-mail: jiaofy@yeah.net

Key words: Children, Aminophylline poisoning, treatment

Received: May 15, 2018; Accepted: May 31, 2018; Published: June 04, 2018 
Table 1. General Information

\begin{tabular}{|c|c|c|c|c|c|c|c|c|c|c|c|c|c|}
\hline $\begin{array}{l}\text { Clinical } \\
\text { manifest ations }\end{array}$ & Feel sick and vomit & anxious & \begin{tabular}{c|} 
Faster \\
heart rate
\end{tabular} & twitch & $\begin{array}{l}\text { Pale and } \\
\text { hair }\end{array}$ & $\begin{array}{l}\text { Shortness } \\
\text { of breath }\end{array}$ & fever & Sweaty & thirst & $\begin{array}{c}\text { Look } \\
\text { forward }\end{array}$ & $\begin{array}{c}\text { Cerebral } \\
\text { palsy }\end{array}$ & $\begin{array}{c}\text { Unconscious } \\
\text { action }\end{array}$ & $\begin{array}{l}\text { hema } \\
\text { turia }\end{array}$ \\
\hline $\begin{array}{l}\text { Number of cases } \\
\text { (Unit: example) }\end{array}$ & $\begin{array}{l}106 \text { cases }(7 \text { cases } \\
\text { with vomit-like } \\
\text { coffee-like contents) }\end{array}$ & 80 cases & 65 cases & 49 cases & 17 cases & 32 cases & 13 cases & 3 cases & 15 cases & 8 cases & 1 case & 11 cases & 2 cases \\
\hline Percentage (\%) & $96.3 \%$ & $72 \%$ & $59 \%$ & $44.5 \%$ & $15.4 \%$ & $29 \%$ & $11.8 \%$ & $2.7 \%$ & $13.6 \%$ & $7.2 \%$ & $0.9 \%$ & $10 \%$ & $1.8 \%$ \\
\hline
\end{tabular}

$37.8{ }^{\circ} \mathrm{C}$ breath: 30 times / heart rate: 128 beats / min blood pressure: $108 / 67 \mathrm{mmHg}$, mentally poor, Shortness of breath, bilateral pupils and other large circles, the presence of light reflex. Soft neck, lips without cyanosis, pharyngeal hyperemia, no abnormalities in heart, lung, abdomen and nervous system examinations. Blood routine: leukocytes $10.37 \times 10^{*}$ / $/ \mathrm{L}$ neutrophils 0.78 lymphocytes 0.14 ; $\mathrm{CRP}>5.0$ $\mathrm{mg} / \mathrm{L}$; bedside ECG: sinus tachycardia, T wave changes. Renal work ion: $\mathrm{K} 3.3 \mathrm{mmol} / \mathrm{L} \mathrm{Na} 34 \mathrm{mmol} / \mathrm{L}$ glucose $6.87 \mathrm{mmol} / \mathrm{L}$; Myocardial enzyme spectrum: CK812U/L. Treatment: Oxygen therapy, antiinfection, and nutrient solution such as supplementation of potassium and nutrient solution were given. After 2 hours, symptoms of the child were relieved. After 5 days, the vital signs were stable and there was no special discomfort. All examinations returned to normal.

\section{Discussion}

Aminophylline is a commonly used anti-asthmatic drug in clinical. It is used in the treatment of bronchial asthma. It has a history of years for the rest of the 70. Due to its more adverse reactions, effective blood concentration [6]. The range is narrow, plus the extensive application of receptor agonists in wheezing diseases. The clinical application of theophylline was once cold. The dose of aminophylline can still play a role in relieving asthma and has a certain anti-inflammatory effect. It is widely used in clinical practice. The individual difference of aminophylline is larger, the sensitivity of aminophylline is higher in children, the dose is not easy to be grasped, and some medical staff are not aware of the side reaction of aminophylline, which causes the moderate accident of aminophylline [7].

\section{The role of aminophylline}

Theophylline is a condensate of theophylline and ethylenediamine, is one of the effective anti-asthmatic drugs for the treatment of asthmatic bronchitis, acute bronchitis, and asthmatic pneumonia, its role is: to ease the bronchi Hemorrhoids, in addition to promote drainage, enhance diaphragm contraction and improve heart and kidney function. Common toxic and side effects: Allergic reactions are manifested as skin eczema, urticaria or asthma, may be associated with erythema multiforme drug eruption [8,9]. Hypersensitivity, often in the process of bolus, sudden restlessness, loss of consciousness, cyanosis of lips, and subsequent respiratory heartbeat; drug poisoning is more common in children with overdose or accidental service, exceeding the maximum amount of treatment $(6 \mathrm{mg} / \mathrm{Kg} / \mathrm{times})$, early Nausea, vomiting, irritability, unconsciousness, thirst, dehydration, and hypothermia; vomiting, prolonged vision, expectoration, coma, high fever and prostration, and epileptic seizures. Can cause death due to inhibition of medulla oblongata; aminophylline can sometimes cause nausea, abdominal discomfort, appetite affected, so it is appropriate to take after meals.

\section{Aminophylline poisoning causes}

The effective plasma concentration of aminophylline is $10-20 \mathrm{mg} / \mathrm{L}$. At this time, the anti-asthmatic effect is the strongest; when it exceeds $20 \mathrm{mg} / \mathrm{L}$, toxicity reaction occurs; if it exceeds $25 \mathrm{mg} / \mathrm{L}$, poisoning may occur. The bioavailability and clearance rate in vivo have large individual differences. Primary hospitals cannot be monitored for blood quality drug concentration because of limited conditions. Therefore, it is difficult to grasp the dose, and if used improperly, it is prone to poisoning. In infants and young children, part of theophylline can be metabolized into caffeine by $\mathrm{N}$-methylation at the 7-position, and the blood-brain barrier is imperfect, and poisoning is more likely to occur.

\section{The rescue process should be noted}

For infants and young children suddenly appear irritated, cry, vomiting more frequently, should be asked medication history, early diagnosis, timely treatment; and according to the specific circumstances of children, symptomatic treatment: such as the application of sedatives, cardiac stimulant, suction Oxygen, fever, respiratory failure or body gram, etc., and should be guarded by a person to observe closely; remove unabsorbed drugs: oral poisoning, such as those who did not vomit within 4 hours after taking (or light vomiting), are Should be gastric lavage, more than 4 hours should be given enema and laxatives, can also be anal administration should wash the intestines, and immediately stop the drug; Diluted absorbed drugs, and promote excretion: mainly with $10 \%-15 \%$ glucose solution intravenously Or intravenous infusion, not only can dilute the toxic and diuretic effects, but also can reduce brain water arsenic. Treatment should pay attention to whether there is heart failure, pulmonary edema. For those who are severely dehydrated, dehydration and electrolyte disturbances should be corrected first. After the dehydration is corrected, high glucose solution is used. Since theophylline is excreted from the kidney, diuretics such as hydrochlorothiazide and furosemide may be used for treatment. Reduce leakage and prevent bleeding: Vitamin $\mathrm{K}$, $\mathrm{C}$ and hormones can play a major role in the treatment of nitrogenbased theophylline poisoning. Do not use ephedrine because of the synergistic effect of ephedrine and theophylline and enhance the toxicity of aminophylline. After animal experiments with excessive aminophylline, a small amount of ephedrine can double the toxicity. It is also improper to use epinephrine [10-12].

\section{Conclusion}

The use of aminophylline should be strictly controlled indications, contraindications and injection dose and usage. Asked about the history of medication. If aminophylline is used within 6 hours, the starting dose should be halved, or the patient must be rescued because of pneumonia. In severe adverse reactions, the use of naloxone can antagonize B-endostatin and block its toxic effects. The use of traditional Chinese medicines such as Angong Niuhuang Pills to stop phlegm and turbulent winds, and blood perfusion and hemodialysis when necessary, is also one of the important and effective means to eliminate the toxic effects of aminophylline. The dosage of aminophylline should be strictly in line with package insert or Chinese Pharmacopoeia. Combination utilization of drugs which would increase the concentration of aminophylline should be avoided.

\section{References}

1. Song XW, Pang DZ (2008) Pediatric clinical medication 100 question. First edition. Beijing: Chemical Industry Press. 
2. State Pharmacopoeia Commission (2005) People's Republic of China Pharmacopoeia.1 notice on drug use in bed (chemical drugs and biological products volume) Beijing: People's hygiene Edition society.

3. Liu Ju, Zhu FL (2004) 36 cases of aminophylline poisoning. Pract Pediat Clin J 19: 322.

4. Feng QY, He Zh L (2007) Cases of infant poisoning caused by aminophylline. Medical Guide 26: 431.

5. National Pharmacopoeia Commission, People's Republic of China Pharmacopoeia (2005) Chemical drugs and biological products volume. Beijing: People's hygiene Edition society.

6. Wang Yu, Ren Ti (1995) Pharmacokinetics of aminophylline in neonates. Chin J Hosp Pharm 15: 534

7. Liu Xiaoman, Jin Wen, Zhang Lei, Liu Jianfei, Jin Gang, et al. (2018) The treatment of arrhythmia caused by aminophylline poisoning in 4 cases. Clin Med Res Pract 3: 24-25.
8. Xu Qiong, Song XW, Wang Y, Zhang HN (2010) Clinical analysis of 9 cases of aminophylline poisoning in preschool children. Chin Pharm 21: 4585-4587.

9. Wang Fei (2015) Diagnosis and treatment of aminophylline poisonin. World's latest medical information digest 15: 132104

10. Xiao Xueqin, Liu YM, LV Hui (2008) The effect of aminophylline poisoning on the level of TNF- alpha and IL-10 and the effect of hemoperfusion on it. Modern Prevent Med 947-949.

11. Shu Hongbin (2012) Large dose of luminal rescue 9 cases of aminophylline poisoning. Chin Pract Med 7: 200

12. Yokoba M, Katagiri M, Ichikawa T, Takakura A, Ishii N, et al. (2015) Aminophylline increases respiratory muscle activity during hypercapnia in humans. Pulm Pharmaco Ther 30: 96-101. [Crossref]

13. Parameswaran K, Belda J, Rowe BH (2000) Addition of intravenous aminophylline to beta2-agonists in adults with acute asthma. Cochrane Database Syst Rev 4: CD002742. [Crossref]

Copyright: (C2018 Jiao F. This is an open-access article distributed under the terms of the Creative Commons Attribution License, which permits unrestricted use, distribution, and reproduction in any medium, provided the original author and source are credited. 